\title{
Parameter Adaptive Terminal Sliding Mode Control of Flexible Coupling Air-Breathing Hypersonic Vehicle
}

\author{
Haibing Chen, ${ }^{1,2}$ Wei Lin, ${ }^{1}$ Tielin Ma $\mathbb{D}^{3}{ }^{3}$ Hengxian Jin, ${ }^{4}$ and Cheng $\mathrm{Xu}^{5}$ \\ ${ }^{1}$ School of Transportation Science and Engineering, Beihang University, Beijing, China \\ ${ }^{2}$ Aircraft/Engine Integrated System Safety Beijing Key Laboratory, Beijing, China \\ ${ }^{3}$ Institute of Unmanned System, Beihang University, Beijing, China \\ ${ }^{4}$ School of Energy and Power Engineering, Beihang University, Beijing, China \\ ${ }^{5}$ Science and Technology on Complex System Control and Intelligent Agent Cooperation Laboratory, Beijing, China \\ Correspondence should be addressed to Tielin Ma; matielin@buaa.edu.cn
}

Received 13 January 2020; Revised 17 June 2020; Accepted 20 June 2020; Published 11 July 2020

Academic Editor: Kenneth M. Sobel

Copyright (C) 2020 Haibing Chen et al. This is an open access article distributed under the Creative Commons Attribution License, which permits unrestricted use, distribution, and reproduction in any medium, provided the original work is properly cited.

\begin{abstract}
The highly nonlinear and coupling characteristics of a flexible air-breathing hypersonic vehicle create great challenges to its flight control design. A unique parameter adaptive nonsingular terminal sliding mode method is proposed for longitudinal control law design of a flexible coupling air-breathing hypersonic vehicle. This method uses adaptive reaching law gain instead of the additional adaptive compensation term to handle the uncertainty to improve robustness. The stability of the close loop system is proved via a Lyapunov way. The longitudinal tracking control law for velocity and angle of attack is designed based on a rigid dynamic model of a flexible air-breathing hypersonic vehicle. A strong coupling model of the same vehicle, considering aerodynamic-scramjet engine-flight dynamic-elastic couplings, is established as the verification platform of the designed control law. The remarkable differences of flight dynamic characteristics between this strong coupling model and the rigid body model can be seen, which mean the controller needs to endure very great uncertainty, unmodeled dynamics, and other types of internal disturbance. Simulation results based on the coupling model demonstrate that the designed control law has good performance and acceptable robustness.
\end{abstract}

\section{Introduction}

A hypersonic vehicle is a kind of aircraft which can fly at more than Mach 5. Compared with conventional aircrafts, hypersonic vehicles have much higher speed and stronger maneuverability. An air-breathing hypersonic vehicle (AHV) is one of the configurations with the most potential in the research and development of hypersonic vehicles. The special designs of AHV, i.e., the high integration of the scramjet propulsion system and airframe and the wave-rider configuration, bring much more nonlinearity and cause strong couplings among the aerodynamic, scramjet propulsion system, flight dynamic, and flexible dynamic. Model uncertainties can arise due to the obvious time-varying flight parameters brought by AHV's high speed and very wide flight envelope, which can also further increase the nonlinearity. Meanwhile, the flexible dynamic cannot be ignored anymore in flight dynamic analy- sis and control design because it has significant impacts on flight characteristics through complex couplings. Consequently, the control design of AHV faces great challenges.

The researches on flight dynamic modelling and control of AHV mainly focus on its longitudinal dynamic. Because of the strong coupling among the aerodynamic, scramjet propulsion system, flight dynamics, and flexible dynamic of AHV, the flight dynamic characteristics of AHV interact with aerodynamic, scramjet propulsion, and fuselage deformation. Chavez and Schmidt made pioneering works in the 1990s [1]. They used Newton's collision theory to estimate hypersonic aerodynamic forces and moment, used onedimensional Rayleigh flow and isentropic flow theory to model the scramjet propulsion system, and established the first AHV flight dynamic model. Besides, this model considers the effect of elastic deformation of the fuselage, and the coupling between aerodynamic and flexible dynamic is 
captured. On the basis of their work, many scholars have made a lot of significant improvements. The well-known work is the longitudinal dynamical modelling finished by Bolender and Doman [2]. They used the oblique shock/expansion wave theory to estimate aerodynamic forces and moment, while the engine model still used the isentropic flow and one-dimensional Rayleigh flow relations, and took into account the influence of elastic deformation of the fuselage. To overcome the difficulty for these physics-based model applications in flight control design, Parker et al. presented some control-oriented models by replacing complex aerodynamic force and scramjet thrust functions in physics-based models with a curve-fitting method [3]. The AHV model which they established describes the coupling among aerodynamic, scramjet propulsion, and flexible dynamic. In fact, the inertia forces generated by the unsteady motion of the aircraft will cause the additional deformation of the fuselage and then affect the aerodynamic and scramjet propulsion system. Such coupling exists among flight dynamic, aerodynamic, scramjet propulsion, and flexible dynamic. In this paper, the flight dynamic model considering the coupling among the aerodynamic, scramjet propulsion, and flexible dynamic is referred to as the weak coupling flexible dynamic model (WCFDM), and the flight dynamic model which also includes the inertial coupling effects between the unsteady motion of AHV and the flexible dynamic is called the strong coupling flexible dynamic model (SCFDM).

Since Bolender and Doman established a general flexible AHV dynamic model that can describe the coupling characteristics among the aerodynamic, flight dynamic, scramjet propulsion, and flexible dynamic of AHV on the basis of Ref. [2], various nonlinear control methods have been applied to the controller design of AHV. In the design of AHV controllers, the robustness and the adaptability are highly important and emphasized because of the dynamic characteristics of AHV, such as strong coupling, nonlinearity, and model uncertainties, as well as physical constraints of control inputs and disturbances.

The control designs of AHV are mostly based on robust control, adaptive control, inversion control, etc. These methods always are combined with each other or with other methods to synthesize or improve to meet the requirements of the control performance of AHV. In Ref. [4], a robust optimal controller design problem is investigated for the longitudinal dynamics of a generic hypersonic vehicle. In the design of the controller, parametric uncertainties, nonlinear and coupling dynamics, unmodeled uncertainties, and external atmospheric disturbances are treated as so-called equivalent disturbances, and a linear time-invariant robust controller is proposed to deal with these problems, which has two parts: an optimal controller to achieve the desired tracking performance and a robust compensator to restrain the influence of the equivalent disturbances. A robust fuzzy disturbance observer-based control design methodology with adaptive bounding is proposed in Ref. [5] for the longitudinal dynamics of a generic hypersonic vehicle using a linear variable parameter model derived from the rigid model by using a functional replacement method. And the stable tracking control of velocity and altitude commands is achieved under the influence of uncertainties and disturbances. An adaptive back-stepping control method considering rudder saturation for the longitudinal control of a hypersonic vehicle is proposed in [6]. Firstly, the linearized model is obtained by using the small perturbation theory. On the basis of the linearized model, the attitude back-stepping control is designed by considering the rudder saturation, and the uncertainties are estimated and compensated for by using the extended state observer. Rollins et al. [7] designed a nonlinear adaptive dynamic inversion control law including a control allocation strategy to track the given flight path angle for a general hypersonic vehicle. The final robustness analysis showed that the controller could offset the effects of time delay and external disturbances and also could deal with the situation of engine inlet failure. A longitudinal inversion flight controller of AHV using nonlinear dynamic inversion in the inner loop and back-stepping method in the outer loop is developed in Ref. [8] to ensure the control performance and the global stability of the system and to restrain the disturbance of uncertainties. In Ref. [9], a fault-tolerant control method based on the back-stepping control is established, which can reduce the control performance degradation when one or more actuators are saturated or faulty, to make the AHV reach a specified steady state. In addition to conventional nonlinear control methods, intelligent control such as fuzzy control and neural network control is also used in the control design of AHV. Since almost all of nonlinear systems can be approached by fuzzy models with any specified accuracy, the application of fuzzy control in the design of AHV controllers has attracted a lot of attention, such as Ref. [10-14]. AHV control design based on neural network control is another research hotspot and has achieved considerable results. Johnson et al. [15] and Bahm et al. [16] have designed a model reference adaptive control system based on neural network for X-33 aircraft in the launch phase and compared it with the conventional gain presetting method in the case of engine failure. With the same control performance, the adaptive control system shows stronger decoupling ability and robustness compared with the gain presetting method. In Ref. [17-19], neural network-based adaptive backstepping controllers are introduced, where nonlinear functions in the AHV dynamic model are approached by various kinds of neural networks. A back-stepping controller with good control performance under strong disturbance is designed using a radial basis function (RBF) neural network in Ref. [20].

In addition to the control methods mentioned above, the sliding mode control (SMC) has also been used in the control design of $\mathrm{AHV}$ because of its robustness and fast response capability. SMC, also known as variable structure control, is a special kind of nonlinear control method. The main difference between SMC and other control strategies is that the structure of the system is not fixed, which can change according to the current situation of the system (such as deviation and its derivatives) in the dynamic process, and therefore makes the system move along the state trajectory defined by the predetermined "sliding mode." Because the sliding mode can be chosen to be independent of the object parameters and disturbances, the sliding mode control has the 
advantages of fast response, insensitivity to the corresponding parameter changes and disturbances, no need of online identification of the system, etc. [21, 22]. As a result of these advantages, the sliding mode control is common in practical applications, and it is also applied to the control design of $\mathrm{AHV}$. Xu et al. [23] put forward an adaptive terminal sliding mode controller for a rigid body linearization model of AHV, in which parameters of the aircraft or aerodynamic parameters are estimated online via an adaptive law, and the desired performance is achieved under the influence of parameter uncertainties. However, the control design does not take into account the influence of flexible dynamic. In Ref. [24], based on linearizing the flexible AHV model, an adaptive sliding mode controller is designed. The controller adjusts one of the parameters of the control law online through the adaptive law and has good performance in tracking altitude and velocity command tracking. But the aerodynamic model used in the control design does not consider the effects of elastic deformation. In order to reduce chattering, a unique smooth function with adjustable parameters is proposed to replace the signum function in the sliding mode control law. However, the stability of the closed-loop system with the new function is not proven. In Ref. [25], a fast adaptive terminal sliding mode controller is designed for a rigid hypersonic vehicle model with unknown upper bound disturbance, which guarantees finite-time convergence of the sliding mode. The upper bound of uncertainties is estimated online by an adaptive law, and the estimation is used as a compensation for the sliding mode control law. Based on the highorder sliding mode theory, Sagliano et al. [26] propose an adaptive sliding mode controller combined with an extended sliding mode observer that reconstructs disturbance online. The controller can be adjusted online under multiple uncertainties to improve performance. Compared with the conventional sliding mode control, the proposed controller has higher tracking accuracy and better robustness for the change of initial conditions or atmospheric parameters. In order to improve the control performance and robustness under the influence of external disturbance and model uncertainties, $\mathrm{Yu}$ et al. [27] develop a continuous high-order finite-time arrival sliding mode control method based on a continuous adaptive second-order sliding mode disturbance observer. Using the fuzzy interval type- 2 system method, a sliding mode controller is designed for the speed and height control of AHV in [28]. The adaptive method is used to adjust parameters of the fuzzy-system-based observer.

In the references mentioned previously, the good performances of the AHV control system are achieved, as well as robustness and disturbance rejection performance. Since the upper bound of system uncertainty is needed for the sliding mode control, adaptive methods or observers are added to the sliding mode control to compensate for the effects of uncertainties in these works. The application of the adaptive method can be divided into three main ways. The first one is to estimate some uncertain parameters of the aircraft online, which mainly deal with the parameter uncertainties. The second one is to estimate the composite disturbances consisting of model uncertainties and disturbances online and compensating for them directly to the control law, and the composite disturbance estimating way is complex and can be regarded as a disturbance observer. The third one is to adjust parameters of the control law by the adaptive method online. In these works, a rigid body or weakly coupling AHV model is mainly used to simulate and verify. In Ref. [24], a coupling model is used for simulation verification, but the aerodynamic calculation of the model does not consider the effect of elastic deformation of the body. Therefore, these models used for simulation verification cannot verify the robustness of the designed controller to model uncertainty including inertia force. The differences between the rigid model and the weak/strong coupling flexible model will be analysed in order to elaborate their characteristics in this paper.

Therefore, in order to design a high-performance adaptive robust nonlinear AHV controller, a novel parameter adaptive terminal sliding mode developed from the conventional nonsingular terminal sliding mode is adopted in this paper. The proposed method uses an adaptive method to adjust the parameter of the control law online to reduce the conservativeness of the system to the bounded uncertainties of the model. Besides, the method replaces the conventional signum function with a smooth function whose parameter is adjustable to reduce the chattering and the aeroelastic vibration that may be induced by the control inputs. With these features, the proposed method achieves the stable tracking control of the AHV velocity and angle of attack commands.

This paper is organized as follows. In Section 2, the longitudinal dynamic model of AHV is described, and the modal characteristics of the rigid AHV model and the weak/strong coupling flexible AHV model are analysed and compared in order to illustrate the differences among them and the fact that the flexible modes and inertial coupling effects should be fully considered in the design of modelling and control. Then, the parameter adaptive terminal sliding mode control method is introduced, and the closed-loop stability of the method is analysed in the Section 3. After that, by dividing the system into two subsystems, the AHV nonlinear rigid body model is used to design the controller. After introducing the controller design, the adaptive terminal sliding mode control method proposed in this paper will be simulated and validated based on the strong coupling flexible AHV model. Finally, conclusions are made.

\section{AHV Model with Aerodynamic-Propulsion- Flight-Flexible Dynamic Coupling}

In this section, an aerodynamic-scramjet propulsion-flightflexible dynamic coupling model of AHV used in this paper is introduced, and its coupling characteristics are analysed. Wave-rider configuration is a relatively mature AHV configuration, and one of the earliest researches was performed by Chavez and Schmidt in 1994 [1]. They established a comprehensive analytical model of the longitudinal dynamics of an AHV using Newtonian impact and quasi one-dimensional Rayleigh flow theory. The first flexible mode was estimated by a complete NASTRAN analysis, and only the coupling between the aerodynamic and the flexible dynamic was considered. Bolender and Doman improved and developed a 
new model using a combination of oblique shock and Prandtl-Meyer expansion theory to calculate the aerodynamic forces and moments and conducted the first principle model by Lagrangian formulation, which was capable of capturing the strong coupling characteristics [2]. The model has a high accuracy for its good grasp of the coupling among the aerodynamic, scramjet propulsion, flight, and flexible dynamic.

Oblique shock and Prandtl-Meyer expansion wave theory are used to calculate aerodynamic forces of the AHV dynamic model in this paper. In addition, engine thrust is calculated by isentropic flow relationship and one-dimensional Rayleigh flow model using liquid hydrogen as fuel. For the slender $\mathrm{AHV}$, it is regarded as two cantilever beams fixed at the centre of mass [29]. The theory of the cantilever beam is used to model the elastic vibration, and then its deformation is obtained to calculate the aerodynamic forces and thrust after deformation.

For AHV, when the aerodynamic forces change, the deformations of the fuselage will change, and consequently, the aerodynamic shape will change. This change will also cause the change of air mass flow and parameters of the air flow entering the scramjet engine, which will lead to the change of the working state of the engine, and the change of the working state of the scramjet engine will cause the change of the aerodynamic force and the deformation angle of the afterward body. This is the so-called coupling effect between aerodynamic, propulsion, and structure. The differential equations of the WCFDM are as follows [30]:

$$
\begin{aligned}
\dot{V} & =\frac{T \cos \alpha-D}{m}-g \sin \gamma, \\
\dot{\alpha} & =q-\frac{L+T \sin \alpha}{m V}+\frac{g \sin \gamma}{V}-\frac{V \cos \gamma}{R_{e}+h}, \\
\dot{q} & =\frac{M_{y}}{I_{y}}, \\
\dot{h} & =V \sin \gamma, \\
\dot{\theta} & =q, \\
\ddot{\eta}_{f} & =-2 \zeta \omega_{f} \dot{\eta}_{f}-\omega_{f}^{2} \eta_{f}+N_{f}, \\
\ddot{\eta}_{a} & =-2 \zeta \omega_{a} \dot{\eta}_{a}-\omega_{a}^{2} \eta_{a}+N_{a},
\end{aligned}
$$

where $\left[V, \alpha, q, h, \theta, \eta_{f}, \dot{\eta}_{f}, \eta_{a}, \dot{\eta}_{a}\right]$ are AHV's velocity, angle of attack, pitching rate, flight altitude, pitching angle, generalized coordinates, and their derivate of modal shape of the forward and the aft beam, respectively, and $\gamma$ is the flight path angle. $T\left(h, V, \alpha, \eta_{f}, \eta_{a}, \delta_{e}, \phi\right), D\left(h, V, \alpha, \eta_{f}, \eta_{a}, \delta_{e}, \phi\right), L(h$, $\left.V, \alpha, \eta_{f}, \eta_{a}, \delta_{e}, \phi\right)$, and $M_{y}\left(h, V, \alpha, \eta_{f}, \eta_{a}, \delta_{e}, \phi\right)$ are the engine thrust, drag, lift, and pitch moment, respectively. They are nonlinear functions of the velocity $V$, the angle of attack $\alpha$, the flight altitude $h$, the generalized coordinates $\eta_{f}$ and $\eta_{a}$, and the control inputs $\delta_{e}$ and $\phi$. These forces and moment used in this paper are calculated by interpolation based on a seven-dimensional interpolation table, which is developed in Reference [31].
One of the important effects considered in this paper is the presence of coupling between the rigid body accelerations and flexible body dynamics. There may exist incorporation of the effects of the rigid body vertical translation rotational effects on the fuselage dynamics. The differential equations of SCFDM capturing inertial coupling effects between the pitching and normal accelerations of the aircraft and the structural dynamics are as follows [2]:

$$
\begin{aligned}
& m \dot{U}+m q W+m g \sin \theta+\dot{q}\left(\lambda_{a} \eta_{a}+\lambda_{f} \eta_{f}\right) \\
& \quad+2 q\left(\lambda_{a} \dot{\eta}_{a}+\lambda_{f} \dot{\eta}_{f}\right)=F x, \\
& m \dot{W}-m q U-m g \cos \theta-q^{2}\left(\lambda_{a} \eta_{a}+\lambda_{f} \eta_{f}\right) \\
& \quad+\lambda_{a} \ddot{\eta}_{a}+\lambda_{f} \ddot{\eta}_{f}=F z, \\
& \left(I_{y}+\eta_{a}^{2}+\eta_{f}^{2}\right) \dot{q}+(\dot{U}+q W)\left(\lambda_{a} \eta_{a}+\lambda_{f} \eta_{f}\right) \\
& \quad+2 q\left(\eta_{a} \dot{\eta}_{a}+\eta_{f} \dot{\eta}_{f}\right)-\psi_{a} \ddot{\eta}_{a}-\psi_{f} \ddot{\eta}_{f}=M y, \\
& \dot{h}=U \sin \theta-W \cos \theta, \\
& \dot{\theta}=q, \\
& \ddot{\eta}_{f}+(\dot{W}-q U) \lambda_{f}-\dot{q} \psi_{f}+2 \zeta \omega_{f} \dot{\eta}_{f}+\left(\omega_{f}^{2}-q^{2}\right) \eta_{f}=N f, \\
& \ddot{\eta}_{a}+(\dot{W}-q U) \lambda_{a}-\dot{q} \psi_{a}+2 \zeta \omega_{a} \dot{\eta}_{a}+\left(\omega_{a}^{2}-q^{2}\right) \eta_{a}=N a .
\end{aligned}
$$

In the above differential equations, $\left[U, W, q, h, \theta, \eta_{f}, \dot{\eta}_{f}\right.$, $\left.\eta_{a}, \dot{\eta}_{a}\right]$ are the velocity along the $x$ body axis, velocity along the $y$ body axis, pitching rate, altitude, pitching angle, generalized modal coordinates, and their derivate of modal shape of the forward and aft beams of the AHV, respectively, and $g$ is the gravity acceleration, $m$ is the mass of the aircraft, and $I_{y}$ is the inertia of the aircraft, The natural frequencies and damping ratios of the fuselage elastic modes of the forward and aft beams of the AHV are $\omega_{i}$ and $\zeta_{i}(i=f, a)$, respectively. $\lambda_{f}=\bar{m}_{f} \int_{0}^{\bar{x}_{f}} \phi(x) d x, \lambda_{a}=\bar{m}_{a} \int_{0}^{\bar{x}_{a}} \phi(x) d x, \psi_{f}=\bar{m}_{f}$ $\int_{0}^{\bar{x}_{f}} x \phi(x) d x$, and $\psi_{a}=\bar{m}_{a} \int_{0}^{\bar{x}_{a}} x \phi(x) d x$ are parameters of inertial coupling terms, and $\phi(x)$ is the mode shape of the fuselage beam. $F_{x}=A_{x}+T-m g \sin \theta+\left(m V^{2} / R_{e}+h\right) \sin \theta$ and $F_{z}=A_{z}+m g \cos \theta-\left(m V^{2} / R_{e}+h\right) \cos \theta$ are forces acting on AHV along the $x$ and $z$ body axes.

For the reason that aerodynamic forces are written in terms of lift and drag, it is necessary to rewrite the body axis equations of motion in terms of stability axes. Based on the definition

$$
\begin{aligned}
\tan \alpha & =\frac{W}{U}, \\
V_{t}^{2} & =U^{2}+W^{2},
\end{aligned}
$$

we can get the differential equations

$$
\begin{gathered}
V_{t}=\frac{\dot{U} U+\dot{W} W}{V_{t}}, \\
\dot{\alpha}=\frac{U \dot{W}-W \dot{U}}{U^{2}+W^{2}} .
\end{gathered}
$$


The SCFDM considers not only the coupling among aerodynamic, scramjet propulsion, and flexible dynamic but also the influence of inertia forces caused by the unsteady motion of the vehicle. When AHV has a certain rotational angular acceleration or oscillation, the fuselage will be affected by the inertia force due to this motion. The inertia forces will cause the change of load on the fuselage, which will lead to deformation angles of the fuselage and change the aerodynamic shape. This will cause the change of aerodynamic forces on the AHV and the change of airflow parameters and air mass flow entering the scramjet engine. Eventually, the working state of the scramjet engine will change. The coupling is a strong coupling among flight, aerodynamic, scramjet propulsion, and flexible dynamic.

The control inputs $\left[\delta_{e}, \phi\right]$ of AHV are the elevator deflection angle and fuel equivalent ratio. They are implicit in the longitudinal dynamic model of AHV through aerodynamic force and moment, thrust, and generalized forces of the forward and aft beams. The speed limit second-order models of the actuators are as follows:

$$
\begin{gathered}
\ddot{\delta}_{e}=\omega_{e}^{2} \delta_{e c}-\omega_{e}^{2} \delta_{e}-2 \omega_{e} \xi_{e} \dot{\delta}_{e}, \\
\ddot{\phi}=\omega_{\phi}^{2} \phi_{c}-\omega_{\phi}^{2} \phi-2 \omega_{\phi} \xi_{\phi} \dot{\phi} .
\end{gathered}
$$

The aerodynamic forces, moment, and thrust of AHV are obviously coupled with the elastic deformation of its forward and aft beams. This leads to the fact that the aerodynamic forces, moment, and thrust of AHV affected by elasticity are very different from those of a rigid body [31]. Therefore, the influence of body elasticity and inertia force cannot be ignored in control law design and verification.

In order to demonstrate the influence of elastic dynamics on the open-loop characteristics of AHV, several typical level flights are selected and trimmed using a rigid body model and flexible models. The trim results of the rigid body and flexible models are shown in Table 1. The angle of attack required for the flexible model is smaller and the elevator deflection is bigger, which is due to the warping of the forward and aft beams of the fuselage. Because the pitching rate and angular acceleration of AHV and the speed and acceleration of elastic vibration of the airframe are all zero, the trim conditions of the two flexible models are the same.

The rigid body AHV model and the two flexible AHV models are linearized at different trim conditions. Table 2 gives the eigenvalues of the linearized dynamics. Similar results can be found in $[2,3]$.

The linearized dynamics shows the significant differences among the three models. The SCFDM demonstrates a new aeroelastic mode and a very different natural frequency and damping ratio of each mode. SCFDM is much more unstable than the rigid body model. For control design in this paper, a short-period mode is crucial and its instability increases almost $40 \%$.

From the analysis above, it can be seen that the flexible modes and inertial force coupling of AHV have a significant impact on its open-loop dynamic characteristics, which should not be ignored in control design. In the following sections, we design controllers only using the rigid body dynamic model, while verifying the whole control law based on SCFDM with additional internal disturbance. The unmeasurable flexible modes and inertial coupling terms are treated as model uncertainties or unmodelled dynamics, which is solved via an adaptive terminal sliding mode method.

\section{Parameter Adaptive Terminal Sliding Mode Control Method}

3.1. Terminal Sliding Surface. Affine nonlinear systems with uncertainties are considered as follows:

$$
\dot{x}=f(x)+g(x) u+d
$$

In Equation (6), $x \in R^{n}$ is the state vector of the system; $u \in R^{m}$ is the control inputs of the system; $f(x)$ and $g(x)$, the certain part of the system, are, respectively, $n \times 1$ and $n$ $\times m$ matrix functions of $x$, and $g(x)$ is reversible; and $d$ denotes the uncertainty of the system, including the modelling error, external disturbance, and parameter uncertainty of the system. It is assumed that $d$ and its derivative terms are bounded, i.e., $\|d\| \leq D_{1},\|\dot{d}\| \leq D_{2}$, in which $D_{1}, D_{2}$ are unknown positive constants.

To avoid the singularity and slow convergence of the conventional terminal sliding mode, the following nonsingular fast terminal sliding mode surface is adopted to accelerate the convergence of tracking error [32]:

$$
\sigma=e+\int_{0}^{t}\left(k_{1} \operatorname{sig}^{\gamma_{1}}(e)+k_{2} \operatorname{sig}^{\gamma_{2}}(e)\right) d \tau, \quad e=x-x_{c},
$$

where $k_{1}, k_{2}>0, \gamma_{1} \geq 1,0<\gamma_{2}<1$ are constants, $e$ is the tracking error, and $x_{c}$ is the reference input.

The function $\operatorname{sig}^{\gamma}(x)$ is defined as the following:

$$
\operatorname{sig}^{\gamma}(x)=|x|^{\gamma} \operatorname{sgn}(x)
$$

This function $\operatorname{sig}^{\gamma}(x)$ is used in the reaching law too, as shown in Section 3.2. $\operatorname{sig}^{\gamma}(x)$ is continuous and smooth, and it replaces the conventional switch function such as sgn $(\cdot)$ to avoid adverse discontinuities in system response and control inputs (e.g., chattering), thereby improving system performance.

When the system reaches the sliding mode surface given by Equation (7), the sliding mode dynamic equation can be obtained as follows:

$$
\left\{\begin{array}{l}
\sigma=0, \\
\dot{\sigma}=\dot{e}+k_{1} \operatorname{sig}^{\gamma_{1}}(e)+k_{2} \operatorname{sig}^{\gamma_{2}}(e)=0 .
\end{array}\right.
$$

That is,

$$
\dot{e}=-k_{1} \operatorname{sig}^{\gamma_{1}}(e)-k_{2} \operatorname{sig}^{\gamma_{2}}(e) .
$$


TABLE 1: Trim points of AHV.

\begin{tabular}{lcc}
\hline Flight condition & Rigid body model & Flexible model \\
\hline $\mathrm{Ma}=6.5, H=24 \mathrm{~km}$ & $\alpha=3.512^{\circ}, \delta_{e}=9.976^{\circ}, \Phi=0.211$ & $\alpha=2.820^{\circ}, \delta_{e}=10.8204^{\circ}, \Phi=0.233, \eta_{f}=6.3035, \eta_{a}=2.290$ \\
$\mathrm{Ma}=8, H=26 \mathrm{~km}$ & $\alpha=3.695^{\circ}, \delta_{e}=10.087^{\circ}, \Phi=0.264$ & $\alpha=2.967^{\circ}, \delta_{e}=10.946^{\circ}, \Phi=0.2935, \eta_{f}=6.3035, \eta_{a}=2.290$ \\
$\mathrm{Ma}=6.5, H=24 \mathrm{~km}$ & $\alpha=3.655^{\circ}, \delta_{e}=10.593^{\circ}, \Phi=0.363$ & $\alpha=2.905^{\circ}, \delta_{e}=11.521^{\circ}, \Phi=0.407, \eta_{f}=7.009, \eta_{a}=3.273$ \\
\hline
\end{tabular}

TABLE 2: Modal characteristics at $\mathrm{Ma}=8, H=26 \mathrm{~km}$.

\begin{tabular}{lccc}
\hline Rigid body dynamic model & WCFDM & SCFDM & Mode \\
\hline-1.25 & -1.25 & -1.732 & Short period \\
1.20 & 1.25 & 1.670 & Short period \\
$-1.15 \times 10^{-5} \pm 3.69 \times 10^{-2} i$ & $1.18 \times 10^{-2} \pm 1.42 \times 10^{-2} i$ & $1.18 \times 10^{-4} \pm 3.02 \times 10^{-2} i$ & Phugoid \\
$-5.97 \times 10^{-4}$ & $-2.20 \times 10^{-2}$ & $-5.329 \times 10^{-4}$ & Altitude \\
& $-0.39 \pm 19.96 i$ & Flex & Flex \\
& $-0.32 \pm 14.93 i$ & $-2.344 \pm 44.9 i$ & Aeroelastic \\
& & $-1.112 \pm 26.045 i$ & Aeroelastic \\
\hline
\end{tabular}

On the sliding mode surface, the tracking error of the system will converge to zero in finite time.

Lemma 1. If both $a_{1}, a_{2}, \cdots a_{n}$ and $1<q<2$ are real numbers, the following inequality always holds [33]:

$$
\left|a_{1}\right|^{q}+\left|a_{2}\right|^{q}+\cdots+\left|a_{n}\right|^{q} \geq\left(a_{1}^{2}+a_{2}^{2}+\cdots a_{n}^{2}\right)^{q / 2} .
$$

Proof. The sliding mode equation, Equation (10), can converge to zero in finite time, and the convergence time satisfies

$$
T \leq \frac{\|e\|^{1-\gamma_{2}}}{\left(1-\gamma_{2}\right) k_{2}} .
$$

The Lyapunov function candidate is chosen as $V=$ $(1 / 2) \sum_{i=1}^{n} e_{i}^{2}$. Introducing Equation (10) in the derivative with respect to time

$$
\begin{aligned}
\dot{V} & =\sum_{i=1}^{n} e_{i} \dot{e}_{i}=\sum_{i=1}^{n} e_{i}\left(-k_{1} \operatorname{sig}^{\gamma_{1}}\left(e_{i}\right)-k_{2} \operatorname{sig}^{\gamma_{2}}\left(e_{i}\right)\right) \\
& =-\sum_{i=1}^{n} k_{1}\left|e_{i}\right|^{\gamma_{1}+1}-\sum_{i=1}^{n} k_{2}\left|e_{i}\right|^{\gamma_{2}+1} \\
& \leq-k_{2} \sum_{i=1}^{n}\left|e_{i}\right|^{\gamma_{2}+1} \\
& \leq-k_{2}\left(\sum_{i=1}^{n} e_{i}^{2}\right)^{\left(\gamma_{2}+1\right) / 2} \\
& =-k_{2}(2 V)^{\left(\gamma_{2}+1\right) / 2} \\
& =-2^{\left(\gamma_{2}+1\right) / 2} k_{2} V^{\left(\gamma_{2}+1\right) / 2} .
\end{aligned}
$$

Then, the tracking error will converge to zero in finite time after reaching the sliding surface. The maximum convergence time can be obtained from Equation (13).

$$
T \leq \frac{\|e(0)\|^{1-\gamma_{2}}}{\left(1-\gamma_{2}\right) k_{2}}
$$

The sliding mode defined by Equation (10) is developed from the conventional terminal sliding mode. When the sliding mode of the system on the sliding mode surface is far away from zero, the convergence rate depends more on the term $k_{1} \operatorname{sig}^{\gamma_{1}}(e)$, while $k_{2} \operatorname{sig} \gamma_{2}(e)$ decides the convergence rate when the sliding mode is close to the zero.

3.2. Parameter Adaptive Terminal Control Law. Conventional reaching law using discontinuous switching function, such as the exponential reaching law shown in Equation (15), results in the discontinuity of the conventional sliding mode control and may cause close loop system chattering.

$$
\dot{\sigma}=-\eta \operatorname{sgn}(\sigma)-k \sigma, \quad \eta>0
$$

Many ways to improve the reaching law have been proposed to avoid the discontinuity and chattering. In this paper, the following sliding mode reaching law is used for control design [34]:

$$
\dot{\sigma}=-l_{1} \sigma-l_{2} \operatorname{sig}^{\eta}(\sigma)
$$

where $l_{1}$ and $l_{2}$ are positive constants and $\eta \in(0,1)$. From Equation (16), it can be determined that the time for $\sigma(t)$ 
to converge from the arbitrary initial state $\sigma(0)$ to terminal sliding surface is

$$
T(\sigma(0))=\frac{\ln \left(1+\left(l_{2} / l_{1}\right)|\sigma(0)|^{1-\eta}\right)}{l_{1}(1-\eta)} .
$$

When the above reaching law is applied, the parameter $\eta$ is related to the convergence rate of the system to the sliding mode surface. The smaller $\eta$ means that the system converges faster when the sliding mode is near the sliding surface. When $\eta$ equals zero, the reaching law Equation (16) is equivalent to $\dot{\sigma}=-l_{1} \sigma-l_{2} \operatorname{sgn}(\sigma)$, which is the traditional exponential reaching law. Therefore, the reaching law Equation (8) can be regarded as an improvement of the conventional exponential reaching law. The sgn $(\cdot)$ function is replaced by the term $\operatorname{sig}^{\eta}(\sigma)$ in the formula to alleviate the chattering phenomenon caused by the direct use of itself.

The derivative with respect to time of the nonsingular fast terminal sliding mode surface defined by Equation (7) is

$$
\begin{aligned}
\dot{\sigma} & =\dot{e}+k_{1} \operatorname{sig}^{\gamma_{1}}(e)+k_{2} \operatorname{sig}^{\gamma_{2}}(e) \\
& =f+g u+d-\dot{x}_{c}+k_{1} \operatorname{sig}^{\gamma_{1}}(e)+k_{2} \operatorname{sig}^{\gamma_{2}}(e) .
\end{aligned}
$$

The nonsingular fast terminal sliding mode control law can be constructed as follows according to Equation (18) and the reaching law Equation (16):

$$
\begin{aligned}
u_{\mathrm{TSMC}}= & -g^{-1}\left[f-\dot{x}_{c}+k_{1} \operatorname{sig}^{\gamma_{1}}(e)+k_{2} \operatorname{sig}^{\gamma_{2}}(e)\right. \\
& \left.+l_{1} \sigma+l_{2} \operatorname{sig}^{\eta}(\sigma)+d\right] .
\end{aligned}
$$

In this control law, the upper bound $D_{1}$ of system uncertainties $d$ is needed, but it is difficult to obtain the determined value in advance in practical application. Therefore, in order to satisfy the accessibility of the sliding mode surface function, an adaptive method can be used to estimate the system uncertainties.

For the system uncertainty $d$ contained in the control law Equation (19), the following adaptive law is used to estimate the uncertainties online, and the online estimation is then used in the control law.

$$
\dot{\hat{d}}=\lambda \sigma
$$

where $\widehat{d}$ is the online estimation of the uncertainties $d$ of the system and $\lambda$ is the learning gain. Then, the final adaptive nonsingular fast terminal sliding mode control law is

$$
\begin{aligned}
u_{\mathrm{ATSMC}}= & -g^{-1}\left[f-\dot{x}_{c}+k_{1} \operatorname{sig}^{\gamma_{1}}(e)+k_{2} \operatorname{sig}^{\gamma_{2}}(e)\right. \\
& \left.+l_{1} \sigma+l_{2} \operatorname{sig}^{\eta}(\sigma)+\hat{d}\right] .
\end{aligned}
$$

The introduction of uncertainty estimation adds an error integral term to the closed-loop system. Excessive learning gain will cause unacceptable overshoot and oscillation in the closed-loop system. To avoid this disadvantage, a parameter adaptive method without the estimate of uncertainties of the system is proposed in this paper, and the control law can be redesigned as

$$
\begin{aligned}
u_{\mathrm{ATSMC}}= & -g^{-1}\left[f-\dot{x}_{c}+k_{1} \operatorname{sig}^{\gamma_{1}}(e)+k_{2} \operatorname{sig}^{\gamma_{2}}(e)\right. \\
& \left.+l_{1} \sigma+\widehat{l}_{2} \operatorname{sig}^{\eta}(\sigma)\right] .
\end{aligned}
$$

The parameter $\hat{l}_{2}$ is an adaptive parameter, which is adjusted online using the adaptive law:

$$
\dot{\hat{l}}_{2}=\mu\|\sigma\|
$$

$\mu>0$ is the parameter of the adaptive law. By adjusting $\widehat{l}_{2}$ online, the chattering caused by the large gain to satisfy the reachability of the sliding mode surface function is avoided, and the upper bound of uncertainty no longer needs to be known.

3.3. Stability Analysation. Using $\kappa$ to represent $(1 / \lambda)\left|\tilde{d}^{T} \dot{d}\right|$, for system Equation (6), it is closed-loop stable, and its sliding mode will converge to the following region when the adaptive nonsingular fast terminal sliding mode control law Equation (21) applied

$$
\|\sigma\| \leq \min \left\{\left(\frac{\kappa}{l_{2}}\right)^{1 /(\eta+1)},\left(\frac{\kappa}{l_{1}}\right)^{1 / 2}\right\} .
$$

Proof. The error between online estimation and actual uncertainties is represented by $\tilde{d}=\hat{d}-d$. The Lyapunov function candidate is defined as follows:

$$
V=\frac{1}{2} \sigma^{T} \sigma+\frac{1}{2 \lambda} \tilde{d}^{T} \tilde{d},
$$

and its derivative with respect to time is:

$$
\begin{aligned}
\dot{V}= & \sigma^{T} \dot{\sigma}+\frac{1}{\lambda} \tilde{d}^{T} \dot{\tilde{d}}=\sigma^{T}\left(f+g u+d-\dot{x}_{c}+k_{1} \operatorname{sig}^{\gamma_{1}}(e)\right. \\
& \left.+k_{2} \operatorname{sig}^{\gamma_{2}}(e)\right)+\frac{1}{\lambda} \tilde{d}^{T} \dot{\tilde{d}} \\
= & -\sigma^{T}\left(l_{1} \sigma+l_{2} \operatorname{sig}^{\eta}(\sigma)+\tilde{d}\right)+\frac{1}{\lambda} \tilde{d}^{T} \dot{\tilde{d}} \\
= & -\sigma^{T} l_{1} \sigma-\sigma^{T} l_{2} \operatorname{sig}^{\eta}(\sigma)-\sigma^{T} \tilde{d}+\frac{1}{\lambda} \tilde{d}^{T}(\dot{\vec{d}}-\dot{d}) \\
= & -\sigma^{T} l_{1} \sigma-\sigma^{T} l_{2} \operatorname{sig}^{\eta}(\sigma)-\frac{1}{\lambda} \tilde{d}^{T} \dot{d} \\
\leq & -l_{1}\|\sigma\|^{2}-l_{2}\|\sigma\|^{\eta+1}+\frac{1}{\lambda}\left|\tilde{d}^{T} \dot{d}\right| .
\end{aligned}
$$

It can be seen from Equation (32) that the sliding mode of the system will converge to the following regions:

$$
\|\sigma\| \leq \min \left\{\left(\frac{\kappa}{l_{2}}\right)^{1 /(\eta+1)},\left(\frac{\kappa}{l_{1}}\right)^{1 / 2}\right\} .
$$

So it can be proven that the system is closed-loop stable by using adaptive nonsingular fast terminal sliding mode 
control law Equation (21), and the sliding mode of the system can converge to the neighbourhood containing the origin point by adjusting the parameters $l_{1}, l_{2}$.

For system Equation (6), it is closed-loop stable when the adaptive nonsingular fast terminal sliding mode control law Equation (22) is applied.

Proof. Assuming that there is a positive number $l_{2 d}>D_{1}$ and $\tilde{l}_{2}=\widehat{l}_{2}-l_{2 d}, \delta=l_{2 d}-D_{1}, \delta>0$. The Lyapunov function candidate is defined as follows:

$$
V_{1}=\frac{1}{2} \sigma^{T} \sigma+\frac{1}{2 \mu} \tilde{l}_{2}^{2}
$$

Its derivative with respect to time is

$$
\begin{aligned}
\dot{V}_{1} & =\sigma^{T} \dot{\sigma}+\frac{1}{\mu} \tilde{l}_{2} \dot{\tilde{l}}_{2}=\sigma^{T}\left(d-l_{1} \sigma-\widehat{l}_{2} \operatorname{sig}^{\eta}(\sigma)\right)+\frac{1}{\mu}\left(\widehat{l}_{2}-l_{2 d}\right) \dot{\widehat{l}}_{2} \\
& \leq\|\sigma\|\|d\|-l_{1}\|\sigma\|^{2}-\widehat{l}_{2}\|\sigma\|^{\eta+1}+\widehat{l}_{2}\|\sigma\|-l_{2 d}\|\sigma\| \\
& =-l_{1}\|\sigma\|^{2}-\widehat{l}_{2}\|\sigma\|^{\eta+1}-\delta\|\sigma\|+\widehat{l}_{2}\|\sigma\| .
\end{aligned}
$$

Letting $\kappa_{1}\|\sigma\|=-\delta\|\sigma\|+\widehat{l}_{2}\|\sigma\|$, the conclusions can be drawn as:

(i) If $\kappa_{1}\|\sigma\|-l_{1}\|\sigma\|^{2}<0$, then $\dot{V}<-\widehat{l}_{2}\|\sigma\|^{\eta+1}$, the sliding mode of the system will converge to the following regions:

$$
\|\sigma\| \leq\left(\frac{\kappa_{1}}{l_{1}}\right)
$$

(ii) If $\kappa_{1}\|\sigma\|-\widehat{l}_{2}\|\sigma\|^{\eta+1}<0$, then $\dot{V}<-l_{1}\|\sigma\|$, the sliding mode of the system will converge to the following regions:

$$
\|\sigma\| \leq\left(\frac{\kappa_{1}}{l \wedge_{2}}\right)^{1 / \eta}
$$

To summarize, sliding mode of the system will converge to the following regions:

$$
\|\sigma\| \leq \min \left\{\left(\frac{\kappa_{1}}{l_{1}}\right),\left(\frac{\kappa_{1}}{l \wedge_{2}}\right)^{1 / \eta}\right\} .
$$

\section{AHV Longitudinal Control Design}

Considering the obvious differences between SCFDM and the rigid model, a controller designed based on the rigid model will face great uncertainties. Disturbances including internal disturbances and aerodynamic parameters should be considered. The control design objective of this paper is to enable AHV to track the given velocity and angle of attack commands stably and accurately with good transmission in the presence of uncertainties and disturbances. The following longitudinal rigid dynamic model of AHV is adopted in the control law design:

$$
\begin{aligned}
& \dot{V}=\frac{T \cos \alpha-D}{m}-g \sin \gamma, \\
& \dot{\alpha}=q-\frac{L+T \sin \alpha}{m V}+\frac{g \sin \gamma}{V}-\frac{V \cos \gamma}{R_{e}+h}, \\
& \dot{q}=\frac{M_{y}}{I}, \\
& \dot{h}=V \sin \gamma, \\
& \dot{\theta}=q .
\end{aligned}
$$

Since the form of the rigid model (33) is different from that of the affine nonlinear system (6), which is the basic form for the control design method proposed in this paper, it is necessary to change the rigid model (33) into (6). This procedure will be further introduced in each control design section.

Because the control inputs $\delta_{e}$ and $\phi$ cannot directly change the angle of attack effectively, they are usually used to generate the pitch moment and change thrust. It is necessary to control the angle of attack via controlling the pitch rate. This means that the pitch rate is a virtual control input in the angle of attack controller. The control structure is divided into two parts, and the control law is designed. The first part is the angle of attack control loop, and the pitch rate command required by the second part is generated according to the angle of attack command. The second part is the speed and pitch rate control loop of AHV, and it obtains the physical control inputs needed by the whole system.

Linearized dynamics show great differences between the rigid body model and the strong coupling model SCFDM. However, the control law design is only based on the rigid body, and the SCFDM is used as the test simulation platform of controllers. There exist very strong uncertainties in the dynamics of velocity, angle of attack, and pitch rate simultaneously in designing the control. In simulation verification, there are additional internal disturbances introduced in the dynamics of the angle of attack and pitch rate to test the robustness of those controllers. From these considerations, we design the whole control structure and all control laws with the proposed adaptive nonsingular terminal sliding mode-based method.

The block diagram of the adaptive nonsingular terminal sliding mode control law of AHV is shown in Figure 1.

4.1. Angle of Attack Loop Control Law Design. The differential equation of the angle of attack in Equation (33) can be rewritten into an affine nonlinear form as follows:

$$
\dot{\alpha}=f_{\alpha}(x)+q
$$

where $f_{\alpha}(x)$ is

$$
f_{\alpha}(x)=-\frac{L+T \sin \alpha}{m V}+\frac{g \sin \gamma}{V}-\frac{V \cos \gamma}{R_{e}+h} .
$$




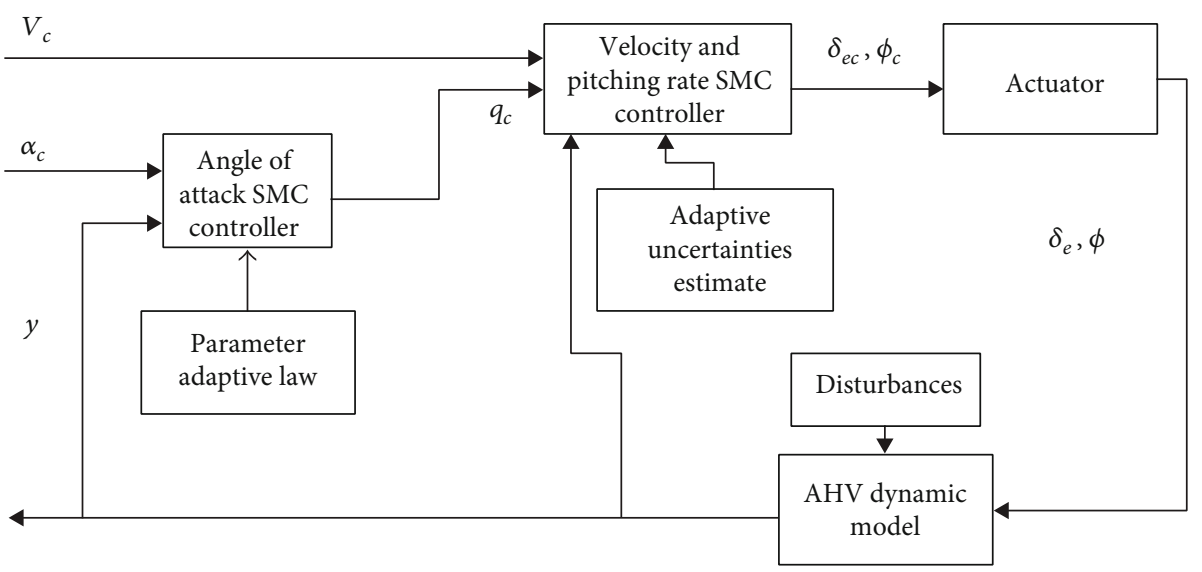

FIgURE 1: AHV adaptive nonsingular terminal sliding mode control block diagram.

The lift $L$ and thrust $T$ are contained in the nonlinear item $f_{\alpha}(x)$, which will be used in derivation of the controller of angle of attack.

The angle of attack tracking error is $e_{\alpha}$, where $\alpha_{c}$ is the command of the angle of attack. According to Equation (34):

$$
\dot{e}_{\alpha}=f_{\alpha}(x)+q-\dot{\alpha}_{c}
$$

The sliding mode surface is taken as the form of Equation (7), and it is

$$
\sigma_{\alpha}=e_{\alpha}+\int_{0}^{t}\left(k_{\alpha 1} \operatorname{sig}^{\gamma_{\alpha 1}}\left(e_{\alpha}\right)+k_{\alpha 2} \operatorname{sig}^{\gamma_{\alpha 2}}\left(e_{\alpha}\right)\right) d \tau
$$

Then, the terminal reaching law Equation (38) is considered,

$$
\dot{\sigma}_{\alpha}=-l_{\alpha 1} \sigma_{\alpha}-l_{\alpha 2} \operatorname{sig}^{\eta_{\alpha}}\left(\sigma_{\alpha}\right)
$$

Considering the sliding surface and the reaching law introduced above, the control law of the angle of the attack loop is

$$
\begin{aligned}
q_{c}= & -f_{\alpha}(x)+\dot{\alpha}_{c}-k_{\alpha 1} \operatorname{sig}^{\gamma_{\alpha 1}}\left(e_{\alpha}\right)-k_{\alpha 2} \operatorname{sig}^{\gamma_{\alpha 2}}\left(e_{\alpha}\right)-l_{\alpha 1} \sigma_{\alpha} \\
& -\widehat{l}_{\alpha 2} \operatorname{sig}^{\eta_{\alpha}}\left(\sigma_{\alpha}\right) .
\end{aligned}
$$

In the control law, parameter $\widehat{l}_{\alpha 2}$ is tuned online by the adaptive law described earlier:

$$
\dot{\hat{l}}_{\alpha 2}=\mu_{\alpha}\left\|\sigma_{\alpha}\right\|
$$

$k_{\alpha 1}, k_{\alpha 2}, \gamma_{\alpha 1}, \gamma_{\alpha 2}, l_{\alpha 1}, \mu_{\alpha}, \eta_{\alpha}$ are parameters of the control law. The range of these parameters and the tuning law can be referred to the analysis in Section 4.3. Notice that $q_{c}$ is the virtual control input to track the angle of attack command, and it is the pitch rate command of the speed and pitch rate control loop at the same time.
4.2. Speed and Pitch Rate Loop Control Law Design. The differential equations of velocity and pitch rate in Equation (33) can be rewritten as follows:

$$
\left\{\begin{array}{l}
\dot{V}=f_{V}(x)+g_{V}(x, u), \\
\dot{q}=g_{q}(x, u),
\end{array}\right.
$$

where $f_{V}(x)=-g \sin (\theta-\alpha), g_{V}\left(x, \delta_{e}, \phi\right)=(T \cos \alpha-D) / m$, and $g_{q}\left(\delta_{e}, \phi\right)=M_{y} / I$. Equation (41) can be further rewritten into the affine nonlinear form approximately, as follows:

$$
\left\{\begin{array}{l}
\dot{V} \approx \tilde{f}_{V}(x)+\tilde{g}_{V \delta_{e}}(x) \delta_{e}+\tilde{g}_{V \phi}(x) \phi, \\
\dot{q} \approx \tilde{f}_{q}(x)+\tilde{g}_{q \delta_{e}}(x) \delta_{e}+\tilde{g}_{q \phi}(x) \phi .
\end{array}\right.
$$

The related functions are as follows:

$$
\begin{aligned}
\tilde{f}_{V}(x) & =f_{V}(x)+g_{V}\left(x, \delta_{e}=0, \phi=0\right), \\
\tilde{f}_{q}(x) & =g_{q}\left(x, \delta_{e}=0, \phi=0\right), \\
\tilde{g}_{V \delta_{e}}(x) & =\frac{g_{V}\left(x, \delta_{e}, \phi\right)-g_{V}\left(x, \delta_{e}=0, \phi\right)}{\delta_{e}}, \\
\tilde{g}_{V \phi}(x) & =\frac{g_{V}\left(x, \delta_{e}, \phi\right)-g_{V}\left(x, \delta_{e}, \phi=0\right)}{\phi}, \\
\tilde{g}_{q \delta_{e}}(x) & =\frac{g_{q}\left(x, \delta_{e}, \phi\right)-g_{q}\left(x, \delta_{e}=0, \phi\right)}{\delta_{e}}, \\
\tilde{g}_{q \phi}(x) & =\frac{g_{q}\left(x, \delta_{e}, \phi\right)-g_{q}\left(x, \delta_{e}, \phi=0\right)}{\phi} .
\end{aligned}
$$

For the convenience of calculation and expression, formula Equation (42) is written as the following form:

$$
\begin{aligned}
& \dot{V}=F_{V}+G_{V} \phi, \\
& \dot{q}=F_{q}+G_{q} \delta_{e},
\end{aligned}
$$


where $F_{V}=\tilde{f}_{V}(x)+\tilde{g}_{V \delta_{e}}(x) \delta_{e}, \quad G_{V}=\tilde{g}_{V \phi}(x), \quad F_{q}=\tilde{f}_{q}(x)+$ $\tilde{g}_{q \phi}(x) \phi$, and $G_{q}=\tilde{g}_{q \delta_{e}}(x)$. The lift $T$, drag $D$, and pitch moment $M_{y}$ are included in these items.

It should be noted that the control design in the following sections is not based on any specific trim status. The complete control input command but not its changing amount about some trim value will be generated directly through the required control force or moment relative to zero control input. Based on this consideration, we chose to calculate the control effectiveness relative to 0, which is Equation (43).

4.2.1. Velocity Controller Design. Based on the affine nonlinear form of the differential equation of velocity Equation (44), the tracking error of velocity command is defined as $e_{V}=V-V_{c}$, then

$$
\dot{e}_{V}=F_{V}+G_{V} \phi-\dot{V}_{c}
$$

Take the terminal sliding mode surface as the form of Formula (7), that is,

$$
\sigma_{V}=e_{V}+\int_{0}^{t}\left(k_{v 1} \operatorname{sig}^{\gamma_{V 1}}\left(e_{V}\right)+k_{v 2} \operatorname{sig}^{\gamma_{V 2}}\left(e_{V}\right)\right) d \tau
$$

Then, considering the terminal reaching law Equation (48),

$$
\dot{\sigma}_{V}=-l_{V 1} \sigma_{V}-l_{V 2} \operatorname{sig}^{\eta_{V}}\left(\sigma_{V}\right) .
$$

As a result, the control law of the velocity controller can be calculated as

$$
\begin{aligned}
\phi_{c}= & -G_{V}^{-1}\left[F_{V}-\dot{V}_{c}+k_{v 1} \operatorname{sig}^{\gamma_{V 1}}\left(e_{V}\right)\right. \\
& \left.+k_{v 2} \operatorname{sig}^{\gamma_{V 2}}\left(e_{V}\right)+l_{V 1} \sigma_{V}+l_{V 2} \operatorname{sig}^{\eta_{V}}\left(\sigma_{V}\right)\right] .
\end{aligned}
$$

$k_{V 1}, k_{V 2}, \gamma_{V 1}, \gamma_{V 2}, l_{V 1}, l_{V 2}, \eta_{V}$ are parameters of the control law. $\phi_{c}$ is the fuel equivalent ratio command that drives AHV to track the velocity command.

4.2.2. Pitching Rate Controller Design. The reference input of the pitch rate control loop is $q_{c}$, which is the pitch rate command generated by the angle of attack controller. Define the pitch rate control tracking error as $e_{q}=q-q_{c}$, then

$$
\dot{e}_{q}=F_{q}+G_{q} \delta_{e}-\dot{q}_{c} .
$$

Taking the terminal sliding mode surface as the form of Equation (7), that is,

$$
\sigma_{q}=e_{q}+\int_{0}^{t}\left(k_{q 1} \operatorname{sig}^{\gamma_{q 1}}\left(e_{q}\right)+k_{q 2} \operatorname{sig}^{\gamma_{q 2}}\left(e_{q}\right)\right) d \tau .
$$

Then, the terminal reaching law Equation (52) is considered,

$$
\dot{\sigma}_{q}=-l_{q 1} \sigma_{q}-l_{q 2} \operatorname{sig}^{\eta_{q}}\left(\sigma_{q}\right) .
$$

As a result, the control law of the pitching rate controller can be calculated as

$$
\begin{aligned}
\delta_{e c}= & -G_{q}^{-1}\left[F_{q}-\dot{q}_{c}+k_{q 1} \operatorname{sig}^{\gamma_{q 1}}\left(e_{q}\right)+k_{q 2} \operatorname{sig}^{\gamma_{q 2}}\left(e_{q}\right)\right. \\
& \left.+l_{q 1} \sigma_{q}+l_{q 2} \operatorname{sig}^{\eta_{q}}\left(\sigma_{q}\right)+\widehat{d}_{q}\right] .
\end{aligned}
$$

In the control law, parameter $\widehat{d}_{q}$ is tuned online by the adaptive law described earlier:

$$
\dot{\hat{d}}_{q}=\lambda_{q} \sigma_{q} .
$$

$k_{q 1}, k_{q 2}, \gamma_{q 1}, \gamma_{q 2}, l_{q 1}, \lambda_{q}, \eta_{q}$ are parameters of the control law.

In order to simplify the derivation operation of the pitch rate command generated by the angle of control law and avoid the problem of "differential expansion" when calculating the differential of the virtual control variable, the pitch rate command is processed using the following first-order low-pass filter:

$$
\dot{q}_{\mathrm{cmd}}=\frac{q_{c}-q_{\mathrm{cmd}}}{\omega_{q}}, \quad q_{\mathrm{cmd}}(0)=q_{c}(0) .
$$

Finally, the control law of the pitch rate controller can be obtained:

$$
\begin{aligned}
\delta_{e c}= & -G_{q}^{-1}\left[F_{q}-\dot{q}_{\mathrm{cmd}}+k_{q 1} \operatorname{sig}^{\gamma_{q 1}}\left(e_{q}\right)\right. \\
& \left.+k_{q 2} \operatorname{sig}^{\gamma_{q 2}}\left(e_{q}\right)+l_{q 1} \sigma_{q}+l_{q 2} \operatorname{sig}^{\eta_{q}}\left(\sigma_{q}\right)+\widehat{d}_{q}\right] .
\end{aligned}
$$

4.3. Control Parameter Determination. The sliding surface parameters $k_{i 1}, k_{i 2}(i=V, \alpha, q)$ determine the convergence rate to zero on the sliding surface of the system. When the error $e_{i}$ is far from the equilibrium point, the convergence rate is mainly affected by $k_{i 1}$. Increasing $\gamma_{i 1}$ properly can also increase the convergence rate. When the error $e_{i}$ is close to the equilibrium point, the convergence rate is mainly affected by $k_{i 2}$, and when $\gamma_{i 2}$ decreases, the convergence rate increases. Note that the integral term $\int_{0}^{t} k_{i 2} \operatorname{sig} \gamma_{i 2}\left(e_{i}\right) d t$ in the sliding surface appears explicitly in the form of $k_{i 2}$ $\operatorname{sig} \gamma_{i 2}\left(e_{i}\right)$ in the control law, and a smaller $\gamma$ makes the function $h(e, \gamma)=\operatorname{sig}^{\gamma}(e)$ closer to the signum function, and the switch would be less smooth consequently. As a result, too large $k_{i 2}$ and too small $\gamma_{i 2}$ will lead to too fast convergence and can cause system chattering.

According to Equation (17), for the parameter design of the reaching law, the smaller parameter $\eta_{i}$ is, the faster the convergence rate of the system is when it is close to the sliding mode surface. When $\eta_{i}$ is 0 , the reaching law Equation (16) is equivalent to the conventional exponential reaching law. The reaching law used in this paper can be regarded as a development of the traditional exponential reaching law. Similarly, because it appears in the control law explicitly, a too small $\eta_{i}$ value will make the function $h(\sigma, \eta)$ close to the signum function and generate the 
chattering phenomenon. Chattering of control variables may excite high frequency vibration of flexible modes.

For the parameters $\lambda$ of the adaptive law, it can be regarded as introducing an error integration feedback with gain $\lambda$. The larger the value of $\lambda$, the faster the adaptive term learns from uncertainty, but too large $\lambda$ will lead to overshoot and oscillation of the system.

Therefore, when choosing the parameters of the controllers, the influence of the above factors should be considered comprehensively. The controller that meets the actual system needs can be obtained by setting the appropriate control parameters.

\section{Simulation and Analysation}

In order to verify effectiveness of the designed control strategy, the adaptive nonsingular fast terminal sliding mode control law is simulated in MATLAB based on the SCFDM, Equation (2). The specific parameters and other related parameters of the aircraft are listed in the appendix.

As a representative case study, the AHV was initially trimmed at the speed of Mach 8 and height of $H=26000 \mathrm{~m}$ . Under this condition, the initial states except height and speed are shown in Table 1 . The parameters of the angle of attack loop controller, velocity controller, and pitching rate controller are selected as shown in Tables 3 and 4 .

The parameters of the second-order actuators, the elevator position limitation and throttle thermally choked limitation, and the rate limitations are shown in Table 5.

The amplitude limit of the elevator deflection angle is a physical limit of elevator deflection, and the upper limit of the throttle amplitude is mainly the thermal resistance limit of the scramjet.

In order to compare the difference between the parameter adaptive method proposed in this paper and the uncertainty estimation method, the two methods are used in the angle of attack control loop in simulation. The response curves of the close loop systems with command are shown in Figure 2.

It can be seen from the response curves that a larger learning gain of the certainty estimation method causes an obvious overshoot, which should be decreased because the AHV's states are sensitive to the angle of attack, while a smaller learning gain causes a longer convergence time. It is because introduction of uncertainty estimation adds an error integral term to the closed-loop system. The system has a smaller overshoot and a faster convergence rate compared with the uncertainty estimation method when using the parameter adaptive method presented.

Compared with the angle of attack control, the pitch rate control emphasizes the response rate more, so the uncertainty estimation method is applied in the pitch rate controller.

When the commands are $\Delta V=50 \mathrm{~m} / \mathrm{s}$ and $\Delta \alpha=1^{\circ}$, the response curves without any parameter perturbation and disturbances are shown in Figure 3.

From the velocity and the angle of attack tracking curves, it can be seen that under the action of the controller, the velocity of AHV can track the velocity step signal accurately in 40-50 seconds and reach the maximum throttle limit at the
TABLE 3: Parameters of the angle of attack controller.

\begin{tabular}{lccc}
\hline Parameter & Value & Parameter & Value \\
\hline$k_{\alpha 1}$ & 0.001 & $l_{\alpha 1}$ & 1 \\
$k_{\alpha 2}$ & 0.001 & $\eta_{\alpha}$ & 0.75 \\
$\gamma_{\alpha 1}$ & 1.01 & $\mu_{\alpha}$ & 55 \\
$\gamma_{\alpha 2}$ & 0.65 & & \\
\hline
\end{tabular}

TABle 4: Parameters of velocity controller and pitching rate controller.

\begin{tabular}{lccc}
\hline Parameter & Value & Parameter & Value \\
\hline$k_{V 1}$ & 0.0001 & $k_{q 2}$ & 0.25 \\
$k_{V 2}$ & 0.005 & $\gamma_{q 1}$ & 1.6 \\
$\gamma_{V 1}$ & 1.001 & $\gamma_{q 2}$ & 0.85 \\
$\gamma_{V 2}$ & 0.2 & $l_{q 1}$ & 0.3 \\
$l_{V 1}$ & 0.1 & $l_{q 2}$ & 4.5 \\
$l_{V 2}$ & 0.1 & $\eta_{q}$ & 0.95 \\
$\eta_{V}$ & 0.5 & $\lambda_{q}$ & 10 \\
$k_{q 1}$ & 0.5 & & \\
\hline
\end{tabular}

TABle 5: Actuator parameters.

\begin{tabular}{lccc}
\hline Parameter & Value & Parameter & Value \\
\hline$\omega_{n \delta_{e}}$ & 80 & $\delta_{e}$ & $-30^{\circ} \leq \delta_{e} \leq 30^{\circ}$ \\
$\zeta_{\delta_{e}}$ & 1 & $\dot{\delta}_{e}$ & $-100^{\circ} / \mathrm{s} \leq \dot{\delta}_{e} \leq 100^{\circ} / \mathrm{s}$ \\
$\omega_{\phi}$ & 6.2 & $\phi$ & $0 \leq \phi \leq 0.55$ \\
$\zeta_{\phi}$ & 1 & $\dot{\phi}$ & $-5 / \mathrm{s} \leq \dot{\phi} \leq 5 / \mathrm{s}$ \\
\hline
\end{tabular}

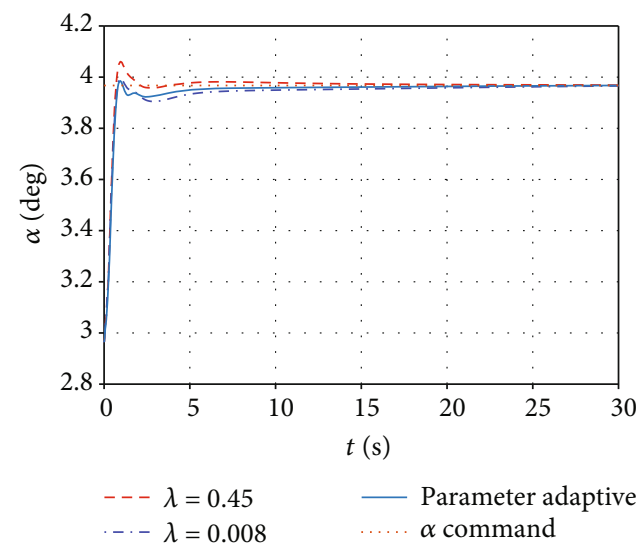

FIGURE 2: Response curves of different methods and parameters.

beginning of the simulation. Due to the coupling of AHV propulsion and aerodynamics and the action of the controller, the overshoot occurs in the first 10 seconds of the process and then gradually converges to the initial value of angle of attack, that is, the reference command of the angle of attack. 

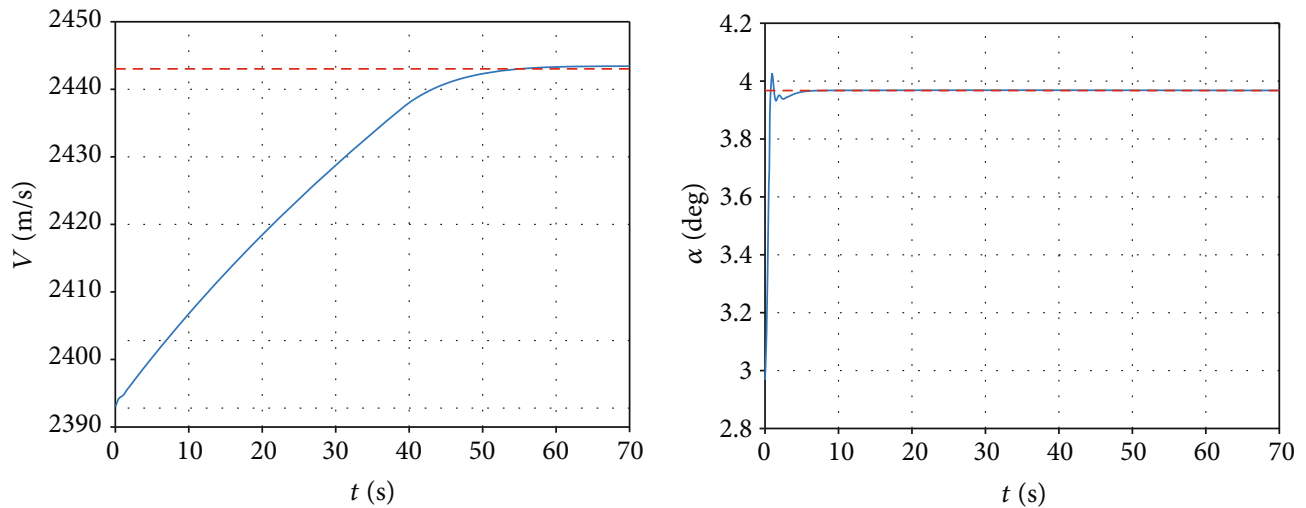

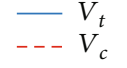

(a)

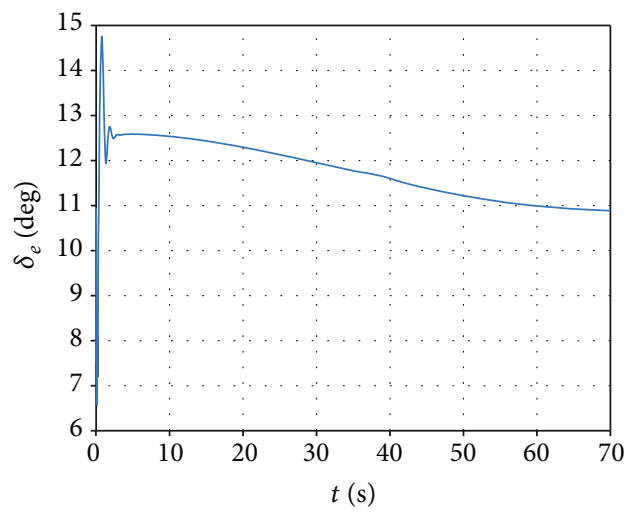

(c)

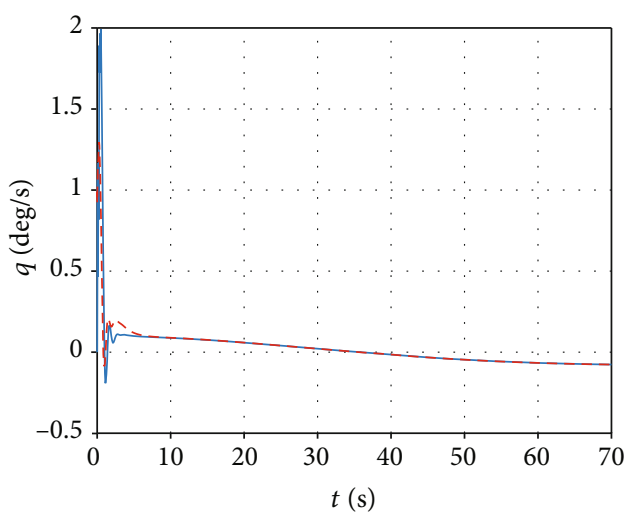

$-q$ $\alpha$
$---\alpha$

(b)

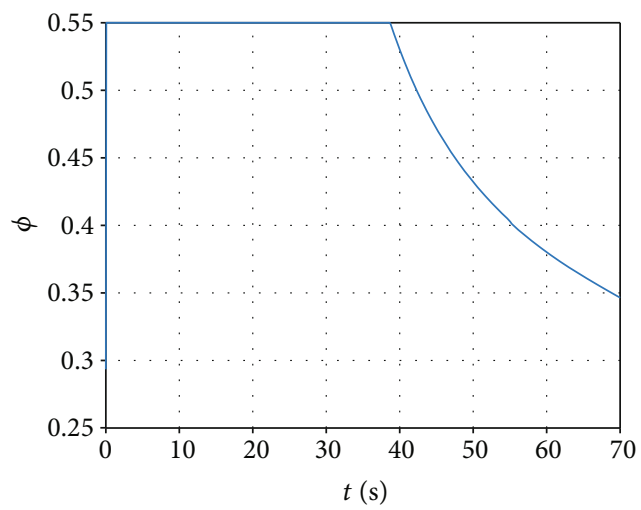

(d)

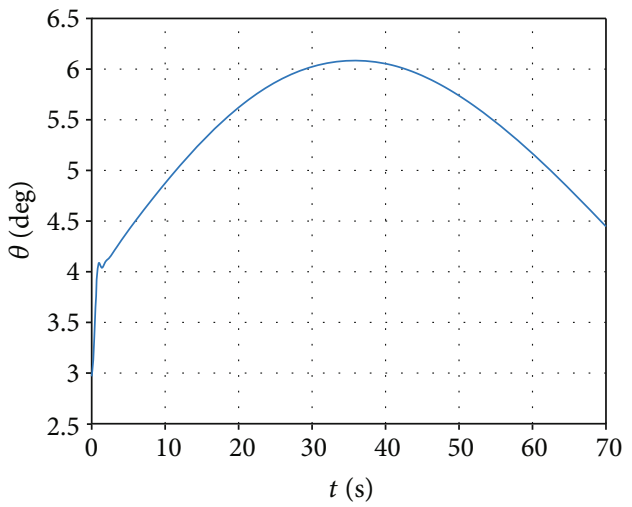

(e)

(f)

Figure 3: Continued. 


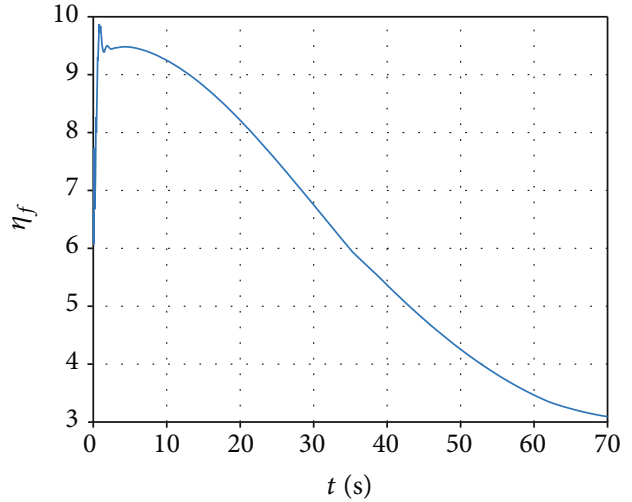

(g)

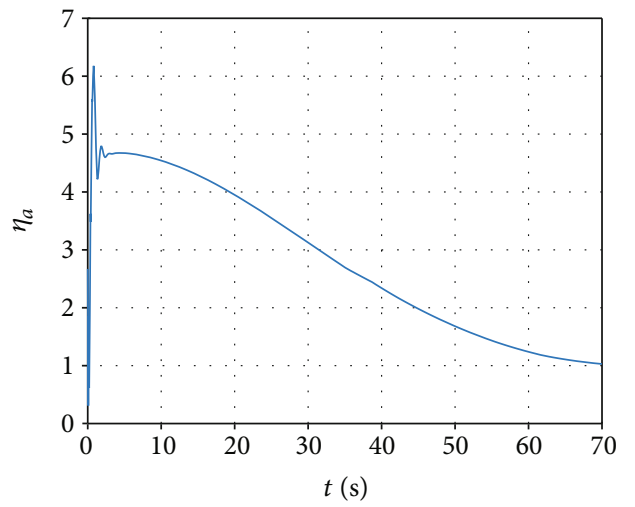

(h)

FiguRE 3: Response of SCFDM without disturbances and perturbation. (a) The response of velocity. (b) The response of the angle of attack. (c) The elevator deflection. (d) The equivalence ratio. (e) The pitch rate. (f) The pitch angle. (g) The generalized modal coordinate of the forward beam. (h) The generalized modal coordinate of the afterward beam.

In order to further verify the control law, more model uncertainties are introduced, such as model perturbation and unmodeled dynamics. Considering a worse situation, $20 \%$ perturbation on aerodynamic parameters is taken into account and additional disturbances are added to the angle of attack control loop and the pitch rate control loop ${ }^{[35]}$, as shown in Equation (57)-(59).

$$
\begin{aligned}
\dot{\alpha} & =F_{\alpha \_ \text {SCFDM }}\left(V, \alpha, q, h, \theta, \eta_{f}, \dot{\eta}_{f}, \eta_{a}, \dot{\eta}_{a}\right)+\Delta \dot{\alpha}, \\
\Delta \dot{\alpha} & =0.02 \sin (0.029 \pi t)\left(\left(^{\circ}\right) / s,\right. \\
\dot{q} & =F_{q \_ \text {SCFDM }}\left(V, \alpha, q, h, \theta, \eta_{f}, \dot{\eta}_{f}, \eta_{a}, \dot{\eta}_{a}\right)+\Delta \dot{q}, \\
\Delta \dot{q} & =\sin (0.0286 \pi t)\left(^{\circ}\right) / s^{2}, \\
T & =(1 \pm 20 \%) T_{\text {nominal }}, \\
D & =(1 \pm 20 \%) D_{\text {nominal }}, \\
M_{y} & =(1 \pm 20 \%) M_{\text {ynominal }},
\end{aligned}
$$

where $F_{\alpha_{\alpha} \text { SCFDM }}$ and $F_{q \_ \text {SCFDM }}$ are the angle of attack and the pitch rate equation of SCFDM, respectively, and $(\cdot)_{\text {nominal }}$ means the nominal aerodynamic force and moment.

The effects of perturbation on aerodynamic parameters are not shown in dynamical Equations (57) and (58) explicitly. They are included in the calculation of the lift $T$, drag $D$, and pitch moment $M_{y}$ of $F_{\alpha_{-} \text {SCFDM }}, F_{q_{-} \text {SCFDM }}$, and velocity equation in SCFDM. The additional disturbances of the angle of attack and pitch rate are directly put in the right side of their dynamical equation as an extra term to represent unknown unmodeled dynamics.

The tracking results of the simulation with commands $\Delta V=50 \mathrm{~m} / \mathrm{s}$ and $\Delta \alpha=1^{\circ}$ are shown in Figure 4. Meanwhile, the tracking results of the control design with the uncertainty estimation method are also shown.

From the simulation results in Figure 4, it can be seen that under the combined influence of parameter perturbation and additional disturbances, the controller designed in this paper can still ensure that the AHV can track the given velocity and angle of attack commands stably. The response time of the angle of attack has only slightly changed compared with that of no disturbance and parameter perturbation, but the convergence time becomes a little bit longer. It can be seen that under the influence of parameter perturbation and system disturbances, the performance of the controller designed in this paper does not show an unacceptable degradation, which reflects the robustness of the method. Compared with the uncertainty estimation method, the parameter adaptive method proposed in this paper has more reasonable transition and performance.

The goal of the control law of this paper is tracking of velocity and angle of attack commands. It guarantees good response of velocity and angle of attack under the effect of coupling. There are no commands of other flight states and the generalized coordinates of the beams in the control structure. The control law assumes that these coordinates are unmeasurable and does not use them. Simulation shows that there is no high frequency and low damping oscillation with high or low amplitude, while this kind of oscillation is the main phenomenon of aeroelastic motion. This demonstrates the advantage of the control design in this paper: no excitation of undesirable aeroelastic motion. However, the control law does not control the coordinates of the beams and other flight states directly when controlling the velocity and angle of attack. So they might show very low frequency and low damping change dominated by other motion modes, which will not end up in the time scale of the finishing velocity and angle of attack command tracking.

It can be seen from the velocity response curves in the above simulation results that the controller accelerates the $\mathrm{AHV}$ at the maximum acceleration at the beginning of the control phase. This causes the fuel equivalence ratio command to exceed the current fuel equivalence ratio saturation value of the AHV for a long time. On the other hand, when the velocity of AHV is close to the velocity command, the controller makes AHV spend a long time in deceleration. The dynamic process of the system is not ideal. The controller should avoid actuator saturation in the control process 


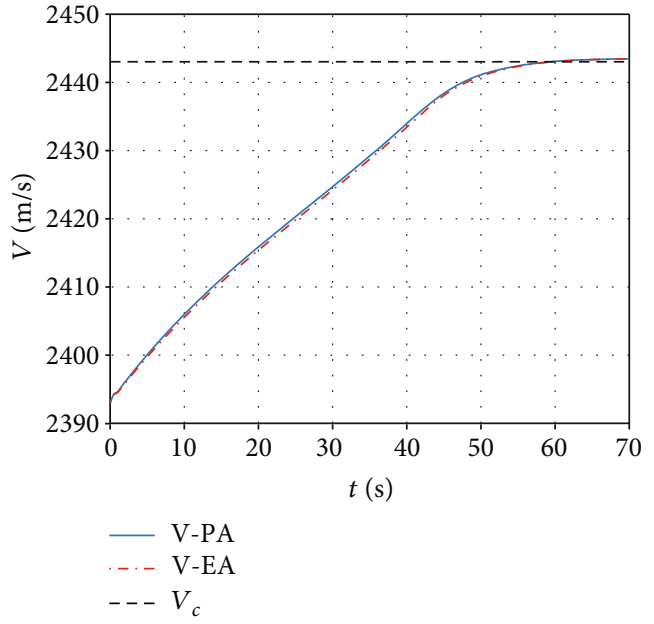

(a)

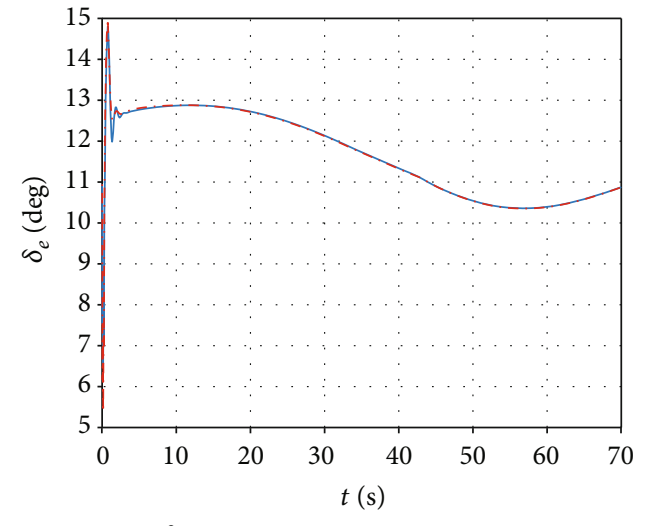

$-\delta_{e}-\mathrm{PA}$
.$--\delta_{e^{-\mathrm{EA}}}$

(c)

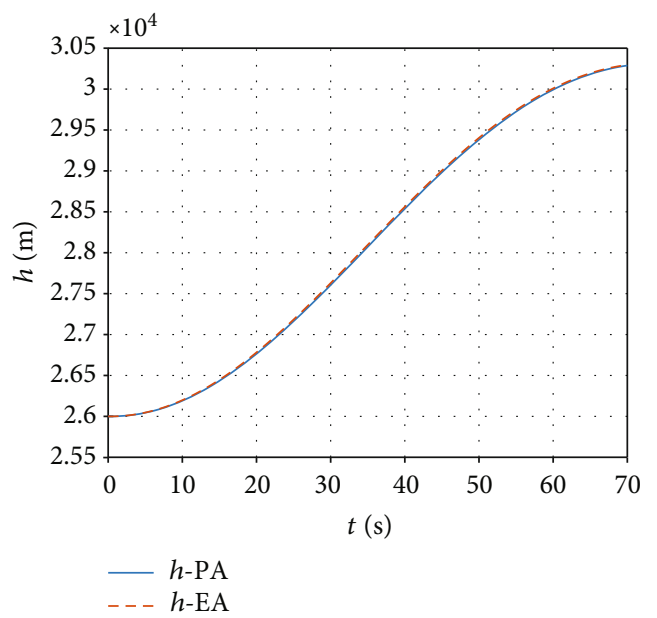

(e)

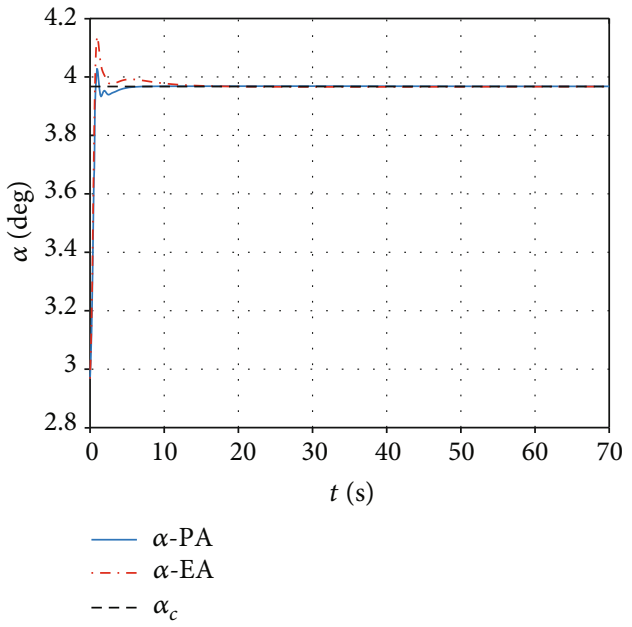

(b)

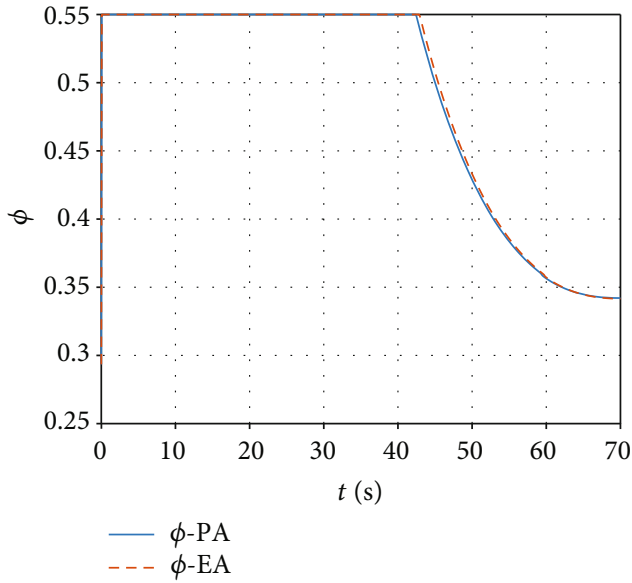

(d)

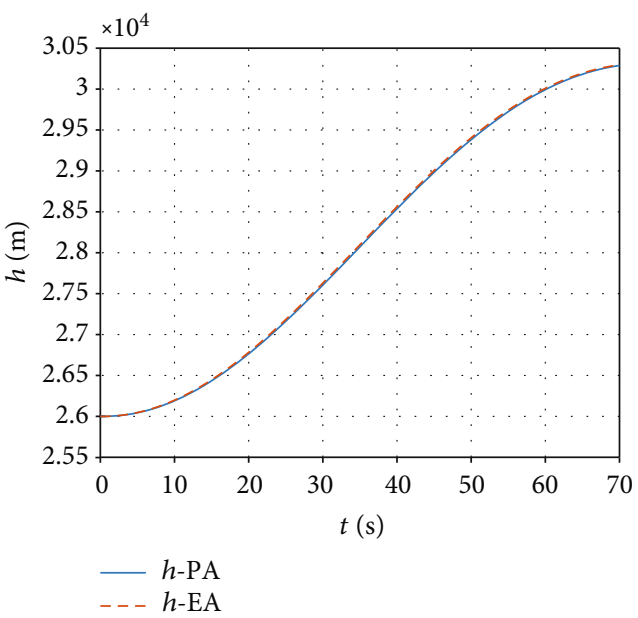

(f)

FIgure 4: Continued. 


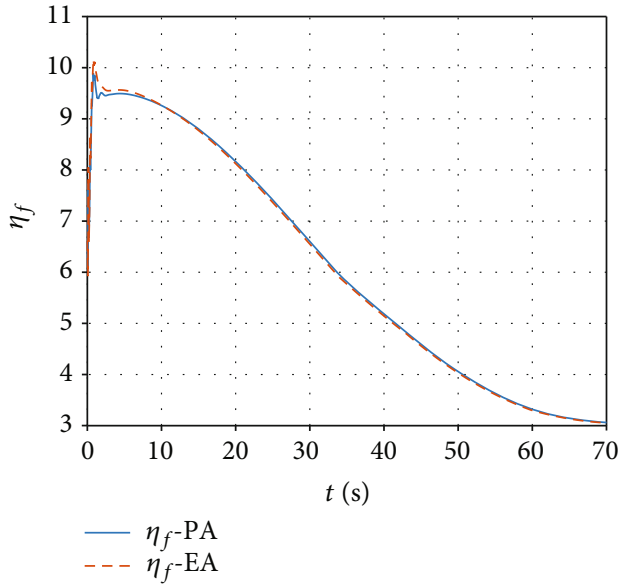

(g)

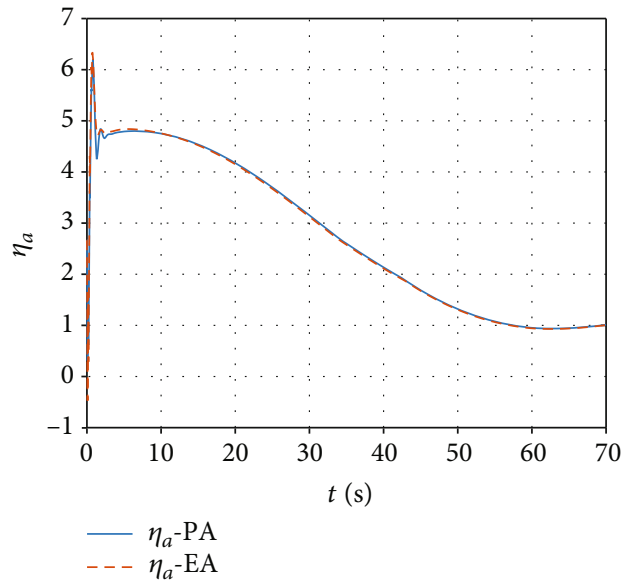

(h)

FIGURE 4: Response of SCFDM with disturbances and perturbation. (a) The response of velocity. (b) The response of the angle of attack. (c) The elevator deflection. (d) The equivalence ratio. (e) The altitude. (f) The pitch angle. (g) The generalized modal coordinate of the forward beam. (h) The generalized modal coordinate of the afterward beam.

and have a reasonable acceleration and deceleration process. This is where improvements need to be made in the follow-up.

\section{Conclusions}

The longitudinal rigid dynamic model and the strong/weak coupling flexible model of an AHV are fully compared and analysed. Integrative design and special geometry make the flexible dynamic of the AHV strongly coupled with flight dynamic, scramjet propulsion, and aerodynamic. The rigid body model cannot capture these coupling characteristics, while the weak coupling model does not consider the influence of inertial force caused by unsteady motion of the AHV. There are great differences among the rigid model and the strong/weak coupling flexible model, which cannot be ignored in control design.

The velocity and angle of attack tracking controller based on the AHV rigid model is designed using the parameter adaptive nonsingular terminal sliding mode control method proposed in this paper. In order to reduce the conservativeness of the sliding mode control to the upper bound of composite disturbance, the controller adjusts the reaching control law gain online via adaptive law rather than the uncertainty estimation whose control performance might be unsatisfactory and achieves stable tracking control when the upper bound of the composite disturbance is unknown. The simulation results based on the strong coupling flexible model show that the designed controllers still have good tracking performance under the influence of model uncertainty, parameter perturbation, and internal disturbance and at the same time avoid exciting the vibration of flexible modes, so as to achieve accurate and stable tracking of velocity and angle of attack commands. The simulation results also show that the proposed method has a smaller overshoot and a faster convergence rate while no additional parameters are required compared with the uncertainty estimation method.

\section{Appendix}

The following is a list of the parameter values for the aircraft configuration: $L=30.48 \mathrm{~m}, L_{f}=14.32 \mathrm{~m}, L_{a}=10.06 \mathrm{~m}$, $L_{n}=6.096 \mathrm{~m}, \tau_{1, u}=3^{\circ}, \tau_{1, l}=6.2^{\circ}, \tau_{2}=14.41^{\circ}, \bar{x}=16.76 \mathrm{~m}$, $\bar{z}=0 \mathrm{~m}, \quad x_{c s}=9.144 \mathrm{~m}, \quad z_{c s}=1.0668, \quad S_{c s}=5.1816 \mathrm{~m}^{2}, \quad h_{i}=$ $1.067 \mathrm{~m}, A_{e}=1.524 \mathrm{~m}^{2}, A_{n}=6.35$, and $A_{d}=1$.

The mass properties of the vehicle are the following: $m=14364 \mathrm{~kg}, \quad \widehat{m}_{f}=385.57 \mathrm{~kg} / \mathrm{m}, \quad \widehat{m}_{a}=575.99 \mathrm{~kg} / \mathrm{m}, I_{y}=$ $2.2241 \times 10^{6} \mathrm{~kg} \cdot \mathrm{m}^{2}$, and $\mathrm{EI}=6.323 \times 10^{8} \mathrm{~N} \cdot \mathrm{m}^{2}$.

\section{Data Availability}

The data used to support the findings of this study are included within the article.

\section{Conflicts of Interest}

The authors declare that there is no conflict of interest regarding the publication of this paper.

\section{Acknowledgments}

We thank Prof. Guodong Ning for his help in starting this research and suggestions to the manuscript. This research is sponsored by the Equipment Research Project Fund under the grant [61403120306].

\section{References}

[1] F. R. Chavez and D. K. Schmidt, "Analytical aeropropulsiveaeroelastic hypersonic-vehicle model with dynamic analysis," Journal of Guidance, Control, and Dynamics, vol. 17, no. 6, pp. 1308-1319, 1994.

[2] M. A. Bolender and D. B. Doman, "Nonlinear longitudinal dynamical model of an air-breathing hypersonic vehicle," Journal of Spacecraft and Rockets, vol. 44, no. 2, pp. 374-387, 2007. 
[3] J. T. Parker, A. Serrani, S. Yurkovich, M. A. Bolender, and D. B. Doman, "Control-oriented modeling of an air-breathing hypersonic vehicle," Journal of Guidance, Control, and Dynamics, vol. 30, no. 3, pp. 856-869, 2007.

[4] H. Liu, D. Liu, and Y. Yu, "Robust optimal controller design for generic hypersonic vehicles," Journal of Aerospace Engineering, vol. 30, no. 4, p. 04017016, 2017.

[5] H. N. Wu, Z. Y. Liu, and L. Guo, "Robust \$L_\{ $\backslash$ bm \infty\}\$Gain Fuzzy Disturbance Observer-Based Control Design With Adaptive Bounding for a Hypersonic Vehicle," IEEE Transactions on Fuzzy Systems, vol. 22, no. 6, pp. 1401-1412, 2014.

[6] Z. Jia, T. Li, and K. Lu, “Adaptive Backstepping Control for Longitudinal Dynamics of Hypersonic Vehicle Subject to Actuator Saturation and Disturbance," Mathematical Problems in Engineering, vol. 2019, 10 pages, 2019.

[7] E. Rollins, J. Valasek, J. A. Muse, and M. A. Bolender, "Nonlinear adaptive dynamic inversion applied to a generic hypersonic vehicle," in AIAA Guidance, Navigation, and Control (GNC) Conference Navigation, \& Control Conference, Boston, MA, August 2013.

[8] Y. B. Liu, "Longitudinal inversion flight control based on backstepping for hypersonic vehicle," Control and Decision, vol. 22, no. 3, pp. 313-317, 2007.

[9] H. An, J. X. Liu, C. H. Wang, and L. G. Wu, “Approximate back-stepping fault-tolerant control of the flexible airbreathing hypersonic vehicle," IEEE/ASME Transactions on Mechatronics, vol. 21, no. 3, pp. 1680-1691, 2016.

[10] X. G. Li and G. Li, "Fuzzy-Approximation-Based Novel BackStepping Control of Flexible Air- Breathing Hypersonic Vehicles with Nonaffine Models," International Journal of Aerospace Engineering, vol. 2019, Article ID 2607195, 19 pages, 2019.

[11] H. N. Wu, S. Feng, Z. Y. Liu, and L. Guo, "Disturbance observer based robust mixed $\mathrm{H}_{2} / \mathrm{H}_{\infty}$ fuzzy tracking control for hypersonic vehicles," Fuzzy Sets and Systems, vol. 306, pp. 118-136, 2017.

[12] H. J. Rong, Z. X. Yang, P. K. Wong, C. M. Vong, and G. S. Zhao, "Self-evolving fuzzy model-based controller with online structure and parameter learning for hypersonic vehicle," Aerospace Science and Technology, vol. 64, pp. 1-15, 2017.

[13] X. Bu, X. Wu, G. He, and J. Huang, "Novel adaptive neural control design for a constrained flexible air-breathing hypersonic vehicle based on actuator compensation," Acta Astronautica, vol. 120, pp. 75-86, 2016.

[14] J. F. Liu, Y. Guo, and S. P. Fang, "Fuzzy model predictive control of flexible air-breathing hypersonic vehicles with unmodeled actuator sensor failures," Advances in Mechanical Engineering, vol. 8, no. 7, Article ID 168781401665594, 2016.

[15] M. D. Johnson, A. J. Calise, and E. N. Johnson, "Evaluation of an adaptive method for launch vehicle flight control," in AIAA Guidance, Navigation, \& Control Conference and Exhibit, Austin, August 2003.

[16] C. Bahm, E. Baumann, J. Martin, D. Bose, R. Beck, and B. Strovers, "The X-43A hyper-X Mach 7 flight 2 guidance, navigation, and control overview and flight test results," in AIAA/CIRA 13th International Space Planes and Hypersonics Systems and Technologies Conference, Capua, Italy, May 2005.

[17] Z. H. Wu, J. C. Lu, and J. P. Shi, "Tracking error constrained robust adaptive neural prescribed performance control for flexible hypersonic flight vehicle," International Journal of Advanced Robotic Systems, vol. 14, no. 1, pp. 1-16, 2017.
[18] G. Zhu and J. Liu, "Neural network-based adaptive backstepping control for hypersonic flight vehicles with prescribed tracking performance," Mathematical Problems in Engineering, vol. 2015, Article ID 591789, 10 pages, 2015.

[19] B. Xu, C. Yang, and Y. Pan, "Global neural dynamic surface tracking control of strict-feedback systems with application to hypersonic flight vehicle," IEEE Transactions on Neural Networks and Learning Systems, vol. 26, no. 10, pp. 25632575, 2015.

[20] X. Bu, X. Wu, Z. Ma, and R. Zhang, "Nonsingular direct neural control of air-breathing hypersonic vehicle via back-stepping," Neurocomputing, vol. 153, pp. 164-173, 2015.

[21] K. D. Young, V. I. Utkin, and U. Ozguner, "A control engineer's guide to sliding mode control," IEEE Transactions on Control Systems Technology, vol. 7, no. 3, pp. 328-342, 2002.

[22] V. I. Utkin, Sliding Modes in Control and Optimization, Springer, Berlin Heidelberg, 1992.

[23] H. J. Xu, M. D. Mirmirani, and P. A. Ioannou, "Adaptive sliding mode control design for a hypersonic flight vehicle," Journal of Guidance, Control, and Dynamics, vol. 27, no. 5, pp. 829-838, 2004.

[24] X. Hu, L. Wu, C. Hu, and H. Gao, "Adaptive sliding mode tracking control for a flexible air-breathing hypersonic vehicle," Journal of the Franklin Institute-Engineering and Applied Mathematics, vol. 349, no. 2, pp. 559-577, 2012.

[25] J. G. Sun, S. M. Song, and G. Q. Wu, "Fault-tolerant track control of hypersonic vehicle based on fast terminal sliding mode," Journal of Spacecraft and Rockets, vol. 54, no. 6, pp. 1304-1316, 2017.

[26] M. Sagliano, E. Mooij, and S. Theil, "Adaptive disturbancebased high-order sliding-mode control for hypersonic-entry vehicles," Journal of Guidance, Control, and Dynamics, vol. 40, no. 3, pp. 521-536, 2017.

[27] P. Yu, Y. B. Shtessel, and C. Edwards, “Adaptive continuous higher order sliding mode control of air breathing hypersonic missile for maximum target penetration," in AIAA Guidance, Navigation, and Control Conference, Kissimmee, Florida, January 2015.

[28] J. L. Gao, R. Y. Yuan, J. Q. Yi, and C. D. Li, “Adaptive interval type-2 fuzzy sliding mode controller design for flexible airbreathing hypersonic vehicles," in 2015 IEEE International Conference on Fuzzy Systems (FUZZ-IEEE), Istanbul, August 2015.

[29] M. Oppenheimer, T. Skujins, M. Bolender, and D. Doman, "A flexible hypersonic vehicle model developed with piston theory," in AIAA Atmospheric Flight Mechanics Conference \& Exhibit, Hilton Head, August 2007.

[30] X. B. Zhang and Q. Zong, "Modeling and analysis of an airbreathing flexible hypersonic vehicle," Mathematical Problems in Engineering, vol. 2014, Article ID 264247, 9 pages, 2014.

[31] H. P. Liu, H. B. Chen, W. Lin, and P. Tang, "Trim and flightelastic coupling characteristics of a flexible air-breathing hypersonic vehicle," in AIAA Scitech 2019 Forum, San Diego, January 2019.

[32] C. Y. Sun, Terminal sliding mode control technology for hypersonic vehicle, The Science Publishing Company, Beijing, China, 2014.

[33] M. P. Aghababa, S. Khanmohammadi, and G. Alizadeh, "Finite-time synchronization of two different chaotic systems with unknown parameters via sliding mode technique," Applied Mathematical Modelling, vol. 35, no. 6, pp. 3080-3091, 2011. 
[34] S. H. Yu, X. H. Yu, and B. Shirinzadeh, "Continuous finitetime control for robotic manipulators with terminal sliding mode," Automatica, vol. 41, no. 11, pp. 1957-1964, 2005.

[35] X. Wang, J. Guo, S. J. Tang, Q. Xu, Y. Ma, and Y. Zhang, "Robust nonsingular terminal sliding mode backstepping control for air-breathing hypersonic vehicles," Acta Aeronautica et Astronautica Sinica, vol. 38, no. 3, article 320287, 2017. 